\title{
Associations between drug burden index, medication appropriateness and patient-reported outcomes in the community pharmacy setting in Malaysia
}

Syed Shahzad Hasan, Annita Shi Chin Liew, David Weng Kwai Chong, Kaeshaelya Thiruchelvam, Zaheer-Ud-Din Babar

Dr. Syed Shahzad Hasan, $P h D$

Corresponding author

University of Huddersfield

Queensgate, HD1 4BX, Huddersfield, UK

Email: $\underline{\text { s.hasan@hud.ac.uk }}$

Ms. Annita Shi Chin Liew, BPharm

International Medical University

No, 126, Jalan Jalil Perkasa 19, Bukit Jalii, Kuala Lumpur, 57000, Malaysia

Email: Annita.ShiChinLiew@student.imu.edu.my

Mr. David Weng Kwai Chong, BPharm, MSc

International Medical University

No, 126, Jalan Jalil Perkasa 19, Bukit Jalii, Kuala Lumpur, 57000, Malaysia

Email: DavidChong@imu.edu.my

Ms Kaeshaelya Thiruchelvam, BPharm, MSc

International Medical University

No, 126, Jalan Jalil Perkasa 19, Bukit Jalii, Kuala Lumpur, 57000, Malaysia

Email: Kaeshaelya@imu.edu.my

Prof. Dr. Zaheer-Ud-Din Babar, PhD

University of Huddersfield

Queensgate, HD1 4BX, Huddersfield, UK

Email: z.babar@hud.ac.uk

Tel: +44 1484471471 


\section{ABSTRACT}

Introduction The increasing complexity of drug regimens over time and the use of multiple medications exposes older patients to a greater risk of receiving inappropriate medications. The study aims to investigate whether drug burden index (DBI) and potentially inappropriate medications (PIMs) were associated with patient-reported health outcomes (PROs) in community-dwelling older adults (aged $\geq 60$ years) in Malaysia.

Methods DBI used to quantify patients' exposure to anticholinergic and sedative medications in a cross-sectional sample of older individuals from the community pharmacy setting in Malaysia. PIMs (based on Beers criteria), potentially inappropriate prescribing (PIP; based on STOPP criteria) and polypharmacy (use of $\geq 5$ concurrent medications) used to quantify exposure to in appropriate medications. PROs were investigated using the Groningen Frailty Indicator (GFI) for frailty, and Older People's Quality of Life-35 (OPQOL) for quality of life (QOL). The adjusted associations between $\mathrm{DBI}$, medication inappropriateness and PROs were analyzed.

Results More than half of the study participants received $\geq 1$ anticholinergic or sedative medication (mean 0.47 participant; range $0.0-2.14$ ). Prevalence of frailty was low; $8.90 \%$ of patients with a mean GFI score of 1.67 (range 1-8) and was significantly and positively correlated with $\mathrm{DBI}(r 0.253 ; p=0.003)$. In multivariate logistic regression, frailty was found to be significantly associated with the odds of receiving DBI-associated medications (odds ratio $1.44 ; 95 \% \mathrm{Cl} 1.02-2.02, p=0.039$ ). Non-significant associations between $\mathrm{DBI}$ and QOL domains, except significant and positive correlation between independence domain of OPQOL and PIPs.

Conclusion The study indicates a significant relationship between drug burden measured by $\mathrm{DBI}$, and frailty measured by GFI. The prescribers managing various medical conditions of older people should consider frailty and other relevant physical health parameters as important health outcomes. 


\section{Introduction}

The ageing population is poised to become one of the most significant social transformations of the $21^{\text {st }}$ century [1]. The World Health Organization predicts an estimated growth of global aging population from 524 million in 2010 to 105 billion in 2050 [2]. Similarly, in Malaysia, as of $2015,8.0 \%$ of the total population consisted of people aged $>60$ years [3]. With the decline in fertility and mortality rates, however, the proportion of older people will expand by 2030 and is predicted to account for $15.0 \%$ of the total population $[1,2]$.

Aging is significantly associated with the development of comorbid chronic conditions, which warrants the use of multiple medications, as per clinical guidelines [3]. Aging is commonly characterized by the emergence of several complex health states, commonly called geriatric syndromes, which highlights unique and common features of health conditions in older people [4]. This includes frailty, urinary incontinence, falls, delirium and pressure ulcers [5-8]. Frailty leads to a state of vulnerability and impaired ability to adapt to external stressors [6], and increases the risk of dependency and adverse events (e.g. cognitive and functional status decline, disabilities, institutionalization and hospitalization) [6]. Frailty coupled with multiple impairments complicate treatment and limit drug choices among older people.

Complexity of drug regimen among older people continues to increase over time, as multiple medications are required to treat a single condition. As a result, polypharmacy is prevalent among older people, with reports of over $45.9 \%$ urban community-dwelling older adults in Malaysia receiving $\geq 5$ medications [9]. Polypharmacy is commonly associated with suboptimal prescribing, which is significantly linked to deterioration in physical functioning status $[10,11]$. Internationally, Gnjidic et al.investigated the association between the Drug Burden Index (DBI), a tool measuring an individual's exposure to sedative and anticholinergic drugs, and the Beers Criteria, a measure of potentially inappropriate drug use, with function in older adults residing in self-care retirement villages [12]. Kojima et al. reviewed the association between frailty and quality of life (QOL) among community-dwelling older people [13]. The literature also vastly 
discusses the improvement in quality of medicines use and patient-reported health outcomes (PROs) via medication reviews for older people [14], with Castelino et al. supporting such improvements when home medicines review services for communitydwelling older people were performed by pharmacists [15]. However, these studies do not focus on the assessment of medication appropriateness and drug burden to optimize functional performance and abilities in frail individuals and those with poor QOL. In addition, there are limited studies investigating such associations among communitydwelling older people in Malaysia $(5,11)$. Hence, the aim of this study is to examine whether DBI and inappropriate medicines use were associated with PROs in communitydwelling older adults in Malaysia.

\section{Methods}

\section{Study design, participants and setting}

This was a cross-sectional study conducted among community-dwelling older people who visited community pharmacies between July and September 2017. This study assessed associations between DBI (i.e. exposure to anticholinergic and sedative medicines), medication inappropriateness and PROs in the community pharmacy setting. Community pharmacies from areas representing different geographic locations were selected because of their convenient accessibility and proximity to the researcher. In total, four community pharmacies, operated by private owners, in Kuala Lumpur and Selangor (both of which are in in peninsular Malaysia) participated in the study.

Participants were included if they had been visiting a community pharmacy, were aged $\geq 60$ years, were mobile, had $\geq 1$ long-term medical condition, had received $\geq 1$ long-term medication, were articulate in English and provided informed consent to participate. An interviewer-administered comprehensive assessment form (CAF) was used to collect demographic data (age, sex, race, marital status, education level, occupation history, number of children and siblings, physical activity level, smoking and alcohol status), QOL, mental health status, frailty status, and medication and medical history, from participants. 
Our investigation complies with the standards of the Helsinki declaration concerning investigation with human subjects. The International Medical University Joint-Committee on Research and Ethics (Project ID: BPI-1-14-(09)2017) provided ethical approval for the study. Permissions from individual community pharmacies were also obtained before data collection. Participants' personal data were stored in a password-protected file accessible only to the researchers. No personal data was disclosed, and the study results are reported as de-identified data.

\section{Assessment of medication inappropriateness}

The inappropriateness of medications was assessed using the Beers criteria (2015), which identifies potentially inappropriate medications (PIMs), and the Screening Tool of Older Persons' potentially inappropriate Prescriptions (STOPP) criteria (2014), which identifies potentially inappropriate prescribing (PIP) $[16,17]$. Both are based on a medicine-to-avoid list by expert consensus $[16,17]$.

The use of medicines use was primarily based on patients' medical records obtained from each participating community pharmacy. The data collected included the name, dose, and dose instructions for all medications used in the 3 months prior to the start date of the study at each facility. Exposure to a PIM, PIP or DBI medication was defined as exposure to an affected medication during the 3-month period.

\section{Assessment of drug burden index}

The DBI was used to quantify individuals' total exposure to anticholinergic and sedative medication, using the following formula [18-20]:

$$
D B I=\frac{D}{D+\delta}
$$

where $D$ is the daily dose taken by the individual within $24 \mathrm{~h}$ (derived from dosing instructions available through medical records), and $\delta$ is the minimum efficacious dose which was approved and registered by the Ministry of Health Malaysia (Formulari Ubat KKM (FUKKM, March 2016). The Malaysian product information and Monthly Index of Medical Specialties (MIMS Malaysia; 2017) were used to identify medications with 
clinically significant anticholinergic and/or sedative effects. Complementary medications, health supplements and medications prescribed on a when required basis were excluded from the DBI calculations (see Supplementary Table for DBI calculation).

\section{Patient-reported health outcomes}

The PROs evaluated were the Groningen Frailty Indicator (GFI), Older People's Quality of Life (OPQOL) inventory, and the Hospital Anxiety and Depression Scale (HADS). The GFI consists of 15 dichotomous items, ranging from a total score of 0 (normal activity without restrictions) to 15 (completely disabled); participants with scores $\geq 4$ were considered frail [21]. We have validated the GFI tool and reported our findings elsewhere [22]. OPQOL was used to assess QOL in 9 domains: overall life, health, social relationships, independence, control over life, freedom, home, and neighborhood, psychological and emotional wellbeing, finance, and religion/ culture [23]. Total OPQOL scores range from 35 (worst possible QOL) to 175 (best possible QOL) [23]. In this study, polypharmacy was defined as $\geq 5$ concurrent medications being dispensed at any one time during the study period, with participants' medication details being obtained from the interview [24]. HADS was used to screen emotional disorders of participants in nonpsychiatric settings by detecting anxious and depressive states, which are the two most frequent distress expressions [25]. It consists of a list of questions, with scores $\geq 11$ for either domain indicating the anxious and depressive states [25].

\section{Sample size and sampling}

The estimated study sample size was 135 , based on the percentage prevalence of Malaysian population aged $\geq 60$ years, with a $95 \%$ confidence level and $4 \%$ margin of error [26]. About 170 older people from community pharmacies in the Kuala Lumpur and Klang Valley regions were approached. Of these, 135 participants who met the inclusion criteria and provided informed consent were included. Before each interview, consent (either verbal or written) was obtained after explaining research objectives to the participants who corresponded to the inclusion criteria. Each interview was conducted in the English language using interviewer-administered CAF. Participants were identified with the help of pharmacist working at the sampled pharmacies. 
Statistical analysis

The data were analyzed using the Statistical Package for Social Sciences (SPSS version $24) ~ \circledR$ with a significance level of 0.05 . The collected data are presented as frequencies, percentages, means and standard deviations. Chi-square test was used to compare PROs and medication-related variables (e.g. frailty and polypharmacy; frailty and $\mathrm{DBI}$ ). Spearman correlation was used to determine the underlying relationship between variables such as DBI, medication appropriateness and PROs. GFI, OPQOL, and HADS scores were changed to binary variables. The median split method was used to split total OPQOL scores (35 to 175) into binary variables (good and poor QOL) [27]. A HADS score of 7 was used to categorize cases and non-cases of depression and anxiety. Unadjusted and adjusted binary logistic regression models were used to examine associations between (1) exposure to a PIM according to the Beers Criteria (yes or no), (2) exposure to a PIP (yes or no) or (3) having a DBI>0 and GFI, OPQOL, and HADS measures (binary variables). The potential confounders were identified based on their association with main variables and based on a priori knowledge [16-20]. We determined variables necessary to control for a confounding effect and that includes age, sex, marital status, number of medications, PROs, and medication-related variables.

\section{Results}

Socio-demographic characteristics of participants

A total of 135 community-dwelling older adults participated in this study. Female and Chinese participants constituted $\approx 46 \%$ and $64 \%$ of the total sampled population, respectively. The mean age of participants was $68.89 \pm 6.14$ years, with the majority of them (94 patients; 69.60\%) being aged 60-69 years. Almost all participants (131; $97.00 \%$ ) were married (Table 1).

Patient-reported health outcomes (PROs)

About $9 \%$ of the participants fell into the frail category (defined as a GFI score of $\geq 4$ ), with a mean score of $1.67 \pm 1.387$ (range 1-8). The mean total OPQOL score was 130.30 
\pm 8.01 (range 109-157) with leisure and activities as the highest domain scored in OPQOL (Table 2).

\section{Use of medicines by the participants}

The mean number of medications per participant was $2.34 \pm 1.37$ (range 1-7). Polypharmacy ( $\geq 5$ medications) was received by $\approx 8 \%(n=11)$ of participants, and $\geq 1$ PIM or PIP by $8.90 \%(n=12)$ and $5.20 \%(n=7)$ of patients, respectively. About $59 \%(n=80)$ of the participants received $\geq 1$ anticholinergic and/or sedative medication included in the DBI (mean 0.47/patient; range: 0-2.14; Table 3).

Drug Burden Index, inappropriate medications and PROs

Exposure to inappropriate medications (including PIMs, PIPs and polypharmacy) occurred in $<10 \%$ of the frail population in the community pharmacy setting. The difference in mean PIMs was statistically significant between frail and non-frail participants ( 0.13 vs $0.03, p=0.001$ ). However, the differences in average PIPs and DBI between frail and non-frail participants were not significant. There was a significant and positive correlation of GFI with number of PIMs $(r 0.255 ; p=0.003)$ and DBI $(r$ 0.253; $p=0.003$ ) in the correlation analysis.

In the total study population, being exposed to $\geq 1$ PIM or PIP according to the Beers and STOPP Criteria was not significantly associated with the total OPQOL score when compared to not being exposed to a PIM or PIP. Among all OPQOL domains, the only significant and positive correlation was between the independence domain of OPQOL and number of PIPs ( $r 0.172 ; p=0.046)$. The correlation analysis also found a significant and positive relationship between DBI and polypharmacy $(r 0.319 ; p=0.001)$.

In the multivariate logistic regression, frailty was found to be significantly associated with the odds of receiving DBI-associated medications (odds ratio1.44; $95 \% \mathrm{Cl}: 1.02-2.02$, $\mathrm{p}=0.039$ ), as depicted in Table 4. Frailty was also significantly associated with exposure to a PIM in the unadjusted model, but became insignificant after adjustment for potential confounders. The OPQOL scores were associated with an increased risk of receiving DBI-associated medications, PIM and PIP, but their associations were not statistically significant. 


\section{Discussion}

The present study examines drug burden and its relationship with medication inappropriateness and PROs in community-dwelling older people in Malaysia. In this community-based study, frail older people were exposed to greater anticholinergic and sedative load than non-frail participants. As a result, frail participants have greater reported medication burden quantified by $\mathrm{DBI}$ compared with non-frail participants. Furthermore, the association remained significant even after adjustment for covariates. The findings from this study were consistent with studies conducted in different countries (USA, Australia and Finland), despite the differences in healthcare access and medication exposure [28-30]. According to some literature, the presence of frailty contributes to the development and progression of multiple chronic comorbidities [31]. As a result, older frail people experience greater complexity in medication regimen, which commonly involves anticholinergic and sedative medications.

Rational prescribing among older people is complex due to lack of medication effectiveness and safety data in this age group [32,33], resulting in a high prevalence of inappropriate medication use among older people. More than half of the older people in this study were exposed to DBI-associated medications and there seemed to be a significant and positive correlation between GFI and medication inappropriateness. The findings from this study are consistent with a retrospective analysis conducted by Castelino et al. in older community-dwelling older individuals prior to a medication review intervention, in which the majority of participants were exposed to DBI-associated medications and one-third had PIMs [15]. This can be explained by age-related changes in pharmacokinetics and pharmacodynamics among older people [34]. Furthermore, the frail elderly people are also known to be more intolerant of medications than their fit counterparts $[34,35]$. They have a diminished ability to adapt to changes with impaired homeostatic mechanisms and decrement in their hepatic metabolism. Indeed, they are expected to portray an exaggerated response to medications [35].

Poorer QOL was also associated with medication inappropriateness. Although there was no statistically significant association identified between both parameters, there was a statistically significant association between medication inappropriateness and the 
physical health domain measured by OPQOL-36. Harrison et al. depicted similar findings in a cross-sectional study with a significant association between higher $\mathrm{DBI}$ and poorer QOL, as well as increasing PIMs and poorer QOL [36]. This can be explained by the nature of inappropriate medication use, which commonly leads to adverse outcomes that affects participants' morbidity and QOL $[37,38]$. In contrast, there was a statistically significant and negative association between medication inappropriateness and dependency. This, however, contradicts several studies that have reported a positive correlation between both parameters. This could be due to the setting of this study as the participants were non-institutionalized individuals with better health status [38, 39] than those in settings such as aged-care facilities, nursing homes and retirements villages $[36,37]$.

Many studies have investigated medication use and high-risk prescribing among community-dwelling older people $[16,24,30,32,33]$. However, to the authors' knowledge, this was the first study in Malaysia (or the region) that investigated the association between medication burden and medication appropriateness. The study has shown statistically significant correlation between medication burden and medication inappropriateness. More participants with $\mathrm{DBI}>0$ than those who were not exposed to $\mathrm{DBI}$-associated medications $(\mathrm{DBI}=0)$ had inappropriate medication use, as shown by the proportions of patients exposed to a PIM (58.33 vs $41.67 \%$ ). PIP (57.14 vs $42.86 \%$ ) or polypharmacy (90.91 vs $9.09 \%)$. Anticholinergics and sedatives were common inappropriate medications prescribed among older people.

There are several strengths to the study. Both implicit and explicit tools were used to measure medication appropriateness. Validated tools were used in this study to investigate medication appropriateness, drug burden and physical health outcomes. However, some limitations could affect the generalizability of the study findings. Firstly, the cross-sectional study design is not suitable to determine the nature of the temporal relationship between medication appropriateness, drug burden and physical function parameters as the exposure and outcome are assessed simultaneously. Secondly, low reliability in self-reported medication use, history and medical conditions may result in an underestimation of medication appropriateness and drug burden. Thirdly, DBI 
calculations use the minimum recommended daily dose, which may vary depending on medication-related pharmacokinetic and pharmacodynamic differences, as well as interindividual variability. This could have caused biasness when determining the pharmacodynamic contribution of individual medications [19]. Fourthly, the use nonprobability sampling (convenience sample) may result in selection bias. Lastly, the unadjusted and adjusted effect sizes are generally biased in most studies except randomized studies. In general, as the sample size increases, the risk of getting biased r-squared would get smaller. We preferred logistic regression because an equivalent statistic to R-squared does not exist (depends on pseudo R-squared values).

In conclusion, the present study found a significant relationship between high-risk prescribing (e.g. higher DBI-associated medications) and PROs such as frailty and QOL. However, exposures to both $\mathrm{DBI}$ and medication inappropriateness were not significantly associated with overall QOL. Medication inappropriateness measured by PIM, PIP and polypharmacy was higher among DBI-exposed participants. The prescribing of medications for managing various medical conditions among older people should consider frailty or other physical health parameters as part of a disease prognosis. Further longitudinal studies investigating the impact of drug burden due to suboptimal prescribing and its association with functional deterioration is important to study the nature of the association.

\section{Compliance with Ethical Standards}

Funding: None.

Conflict of interest: All authors declare that they have no conflicts of interest.

\section{Acknowledgments}

We thank the International Medical University and the management of community pharmacies for the support during this study and extend our appreciation to all participants who took part in the study. 


\section{References}

1. World Population Ageing. New York: Department of Economic and Social Affairs of United Nations; 2015.

2. Global Health and Aging. World Health Organization: National Institute of Aging, National Institutes of Health; 2011 Oct.

3. Zawawi R. Active ageing in Malaysia. In: The second meeting of the committee on international cooperation on active ageing Malaysia. 2013.

4. Department of Statistics Malaysia Official Portal [Internet]. Available from: https://www.statistics.gov.my/

5. Hasan SS, Kow CS, Verma RK, et al. An evaluation of medication appropriateness and frailty among residents of aged care homes in Malaysia. Medicine (Baltimore). 2017;96(35): e7929. doi: 10.1097/MD.0000000000007929.

6. Inouye SK, Studenski S, Tinetti ME, et al. Geriatric syndromes: clinical, research, and policy implications of a core geriatric concept. J Am Geriatr Soc. 2007;55(5):780-91.

7. Basic D, Shanley C. Frailty in an older inpatient population: using the clinical frailty scale to predict patient outcomes. J Aging Health. 2015;27(4):670-85.

8. Fried LP, Tangen CM, Walston J, et al. Frailty in older adults: evidence for a phenotype. J Gerontol A Biol Sci Med Sci. 2001;56(3):M146-56.

9. Walston J, Hadley EC, Ferrucci L, $t$ al. Research agenda for frailty in older adults: toward a better understanding of physiology and etiology: summary from the American Geriatrics Society/National Institute on Aging Research Conference on Frailty in Older Adults. J Am Geriatr Soc. 2006;54(6):991-1001.

10. Eeles EMP, White SV, O'Mahony SM, et al. The impact of frailty and delirium on mortality in older inpatients. Age Ageing. 2012;41(3):412-6.

11. Lim LM, McStea M, Chung WW, et al. Prevalence, risk factors and health outcomes associated with polypharmacy among urban community-dwelling older adults in multiethnic Malaysia. PLOS ONE. 2017;12(3):e0173466.

12. Gnjidic D, Le Couteur DG, Abernethy DR, et al. Drug Burden Index and Beers criteria: impact on functional outcomes in older people living in self-care retirement villages. J Clin Pharmacol. 2012;52(2):258-65.

13. Kojima G, lliffe $S$, Jivraj $S$, et al. Association between frailty and quality of life among community-dwelling older people: a systematic review and meta-analysis. J Epidemiol Community Health. 2016;70(7):716-21.

14. Thiruchelvam K, Hasan SS, Wong PS, et al. Residential aged care medication review to improve the quality of medication use: a systematic review. J Am Med Dir Assoc. 2017;18(1):87.e1-87.e14.

15. Castelino RL, Hilmer SN, Bajorek BV, et al. Drug Burden Index and potentially inappropriate medications in community-dwelling older people: the impact of Home Medicines Review. Drugs Aging. 2010;27(2):135-48.

16. O'Mahony D, O'Sullivan D, Byrne S, et al. STOPP/START criteria for potentially inappropriate prescribing in older people: version 2. Age Ageing. 2015. 1;44(2):213-8. 
17. American Geriatrics Society 2015 Beers Criteria Update Expert Panel. American Geriatrics Society 2015 Updated Beers Criteria for potentially inappropriate medication use in older adults. J Am Geriatr Soc. 2015;63(11):2227-46.

18. Hilmer SN, Mager DE, Simonsick EM, et al. A drug burden index to define the functional burden of medications in older people. Arch Intern Med. 2007;167(8):781-7.

19. Kouladjian L, Gnjidic D, Chen TF, Mangoni AA, Hilmer SN. Drug Burden Index in older adults: theoretical and practical issues. Clin Interv Aging. 2014 Sep 9;9:1503-15.

20. Hilmer SN, Kouladjian O'Donnell L, Gnjidic D. The drug burden index: a tool to assess the functional burden of medications in older adults. Innov Aging. 2017;1(Suppl 1):1242.

21. Steverink N, Slaets JPJ, Schuurmans H, et al. Measuring frailty: Developing and testing the GFI (Groningen frailty indicator). Gerontologist. 2001;41:236-7

22. Hasan SS, Kow CS, Thiruchelvam K, et al. An evaluation of the central nervous system medication use and frailty among residents of aged care homes in Malaysia. Neuroepidemiology. 2017;49(1-2):82-90

23. Bowling A. The Psychometric Properties of the Older People's Quality of Life Questionnaire, compared with the CASP-19 and the WHOQOL-OLD. Curr Gerontol Geriatr Res. 2009;2009:e298950.

24. Gnjidic D, Hilmer SN, Blyth FM, et al. Polypharmacy cutoff and outcomes: five or more medicines were used to identify community-dwelling older men at risk of different adverse outcomes. J Clin Epidemiol. 2012;65(9):989-95.

25. Zigmond AS, Snaith RP. The hospital anxiety and depression scale. Acta Psychiatr Scand. 1983;67(6):361-70.

26. Department of Statistics. Population statistics [Internet]. Putrajaya: Population and Demographic Statistics Division; 2015. [Internet]. Available from: https://www.statistics.gov.my/dosm/uploads/files/3_Time\%20Series/Malaysia_Time_Se ries_2015/22Perangkaan_Penduduk.pdf

27. Hasan SS, Thiruchelvam K, Ahmed SI, et al. Relation between mental health-related variables and glycemic control in Malaysian women with type 2 diabetes mellitus (T2DM). Int J Diab Develop Countries 2014;35(3):211-8

28. Woods NF, LaCroix AZ, Gray SL, et al. Frailty: emergence and consequences in women aged 65 and older in the Women's Health Initiative Observational Study. J Am Geriatr Soc. 2005;53(8):1321-30.

29. Gnjidic D, Cumming RG, Le Couteur DG, et al. Drug Burden Index and physical function in older Australian men. Br J Clin Pharmacol. 2009;68(1):97-105.

30. Gnjidic D, Bell JS, Hilmer SN, et al. Drug Burden Index associated with function in community-dwelling older people in Finland: a cross-sectional study. Ann Med. 2012;44(5):458-67.

31. Murad K, Kitzman DW. Frailty and multiple comorbidities in the elderly patient with heart failure: implications for management. Heart Fail Rev. 2012;17(4-5):581-8.

32. Wilson NM, Hilmer SN, March LM, et al. Associations between Drug Burden Index and falls in older people in residential aged care. J Am Geriatr Soc. 2011;59(5):875-80. 
33. Dedhiya SD, Hancock E, Craig BA, et al. Incident use and outcomes associated with potentially inappropriate medication use in older adults. Am J Geriatr Pharmacother. 2010;8(6):562-70.

34. Cusack BJ, Vestal RE. Clinical pharmacology: special considerations in the elderly. In: The practice of geriatrics. Philadelphia: WB Saunders; 1986.

35. Woodhouse KW, Williams FM, Wynne $\mathrm{H}$, et al. Phase I drug metabolism and ageing. In: Ageing in liver and gastrointestinal tract: Falk Foundation Symposium No 47. Lancaster: MTP Press; 1988.

36. Harrison SL, Kouladjian O'Donnell L, Bradley CE, et al. Associations between the Drug Burden Index, potentially inappropriate medications and quality of life in residential aged care. Drugs Aging. 2018;35(1):83-91.

37. Maclagan LC, Maxwell CJ, Gandhi S, et al. Frailty and potentially inappropriate medication use at nursing home transition. J Am Geriatr Soc. 2017;65(10):2205-12.

38. Franic DM, Jiang JZ. Potentially inappropriate drug use and health-related quality of life in the elderly. Pharmacotherapy. 2006;26(6):768-78.

39. Nordin Olsson I, Runnamo R, Engfeldt P. Medication quality and quality of life in the elderly, a cohort study. Health Qual Life Outcomes. 2011;9:95. 
1 Socio-demographic parameters of participants $(n=135)$

\begin{tabular}{l|c}
\hline \multicolumn{1}{l}{ Variables } & No. of pts $(\%)$ \\
\hline Age $^{\mathbf{a}}$ & $94(69.60)$ \\
\hline $60-69$ years & $34(25.20)$ \\
\hline $70-79$ years & $7(5.20)$ \\
\hline$\geq 80$ years & $62(45.90)$ \\
\hline Sex & $73(54.10)$ \\
\hline Female & $87(64.40)$ \\
\hline Male & $23(17.00)$ \\
\hline Race & $24(17.80)$ \\
\hline Chinese & $131(97.00)$ \\
\hline Malay & $4(3.00)$ \\
\hline Indian & \\
\hline Marital status & \\
\hline Married & \\
\hline \multicolumn{2}{|c}{ Single } \\
\hline Mean age 68.89 years $\pm 6.14($ SD) \\
\hline Pts participants, $S D$ standard deviation \\
\hline
\end{tabular}

Table 2: Patient-reported health outcomes of participants $(n=135)$

\begin{tabular}{|c|c|c|c|}
\hline Variable & Value & Minimum & Maximum \\
\hline \multicolumn{4}{|l|}{ Groningen Frailty Index scores (possible range: $0-15$ ) } \\
\hline Mean score \pm SD & $1.67 \pm 1.39$ & 1 & 8 \\
\hline No. of non-frail pts (score <4) $(\%)$ & $123(91.10)$ & & \\
\hline No of frail pts $($ score $\geq 4)(\%)$ & $12(8.90)$ & & \\
\hline \multicolumn{4}{|c|}{ Hospital Anxiety and Depression Scale scores (possible range: $0-42$ ) } \\
\hline Mean total score \pm SD & $5.49 \pm 6.83$ & 0 & 35 \\
\hline Mean anxiety score (SD) & $3.90 \pm 4.54$ & 0 & 21 \\
\hline Mean depression score \pm SD & $1.59 \pm 2.67$ & 0 & 14 \\
\hline No. of pts without anxiety (score $0-7)(\%)$ & $108(80.00)$ & & \\
\hline No. of pts with anxiety (score 8-21) (\%) & $27(20.00)$ & & \\
\hline No. of participants without depression (score $0-7)(\%)$ & $130(96.30)$ & & \\
\hline No of pts with depression (score 8-21) (\%) & $5(3.70)$ & & \\
\hline
\end{tabular}

OPQOL scores (possible range: $35-175$ )

\begin{tabular}{l|c|c|c}
\hline Mean total score \pm SD & $130.30 \pm 8.01$ & 109 & 157 \\
\hline No. of pts with good OPQOL (\%) & $61(45.20)$ & & \\
\hline No. of pts with poor OPQOL (\%) & $74(54.80)$ & \multicolumn{2}{l}{} \\
\hline OPQOL domain scores \pm SD & $15.52 \pm 2.36$ & 8 & 20 \\
\hline Life overall (possible range: $4-20)$ & $15.34 \pm 2.01$ & 10 & 20 \\
\hline Health (possible range: $4-20)$ & $15.94 \pm 0.62$ & 14 & 18 \\
\hline Social relationships (possible range: $5-25)$ & $16.09 \pm 1.27$ & 11 & 20 \\
\hline Independence, control over like, freedom (possible range: $4-20)$ & $15.90 \pm 1.55$ & 12 & 20 \\
\hline Home and neighborhood (possible range: $4-20)$ & $14.22 \pm 1.28$ & 11 & 20 \\
\hline Psychological and emotional well-being (possible range: $4-20)$ & $16.04 \pm 2.40$ & 8 & 20 \\
\hline Financial circumstances (possible range: $4-20)$ & $21.25 \pm 1.94$ & 15 & 25 \\
\hline Leisure and activities (possible range: $6-30)$ & &
\end{tabular}

OPQOL Older Peoples' Quality of Life version 36, pts participants, $S D$ standard deviation 
